\title{
Novel multiplex technology for diagnostic characterization of rheumatoid arthritis
}

\author{
Piyanka E Chandra ${ }^{1,2}$, Jeremy Sokolove ${ }^{1,2}$, Berthold G Hipp ${ }^{3}$, Tamsin M Lindstrom ${ }^{1,2}$, James T Elder ${ }^{4}$, \\ John D Reveille ${ }^{5}$, Heike Eberl ${ }^{3}$, Ursula Klause ${ }^{3}$ and William H Robinson ${ }^{1,2^{*}}$
}

\begin{abstract}
Introduction: The aim of this study was to develop a clinical-grade, automated, multiplex system for the differential diagnosis and molecular stratification of rheumatoid arthritis (RA).

Methods: We profiled autoantibodies, cytokines, and bone-turnover products in sera from 120 patients with a diagnosis of RA of $<6$ months' duration, as well as in sera from 27 patients with ankylosing spondylitis, 28 patients with psoriatic arthritis, and 25 healthy individuals. We used a commercial bead assay to measure cytokine levels and developed an array assay based on novel multiplex technology (Immunological Multi-Parameter Chip Technology) to evaluate autoantibody reactivities and bone-turnover markers. Data were analyzed by Significance Analysis of Microarrays and hierarchical clustering software.
\end{abstract}

Results: We developed a highly reproducible, automated, multiplex biomarker assay that can reliably distinguish between RA patients and healthy individuals or patients with other inflammatory arthritides. Identification of distinct biomarker signatures enabled molecular stratification of early-stage RA into clinically relevant subtypes. In this initial study, multiplex measurement of a subset of the differentiating biomarkers provided high sensitivity and specificity in the diagnostic discrimination of RA: Use of 3 biomarkers yielded a sensitivity of $84.2 \%$ and a specificity of $93.8 \%$, and use of 4 biomarkers a sensitivity of $59.2 \%$ and a specificity of $96.3 \%$.

Conclusions: The multiplex biomarker assay described herein has the potential to diagnose RA with greater sensitivity and specificity than do current clinical tests. Its ability to stratify RA patients in an automated and reproducible manner paves the way for the development of assays that can guide RA therapy.

\section{Introduction}

Rheumatoid arthritis (RA) is a systemic inflammatory condition characterized by polyarthritis of presumed autoimmune etiology. Although the production of autoantibodies against synovial antigens and an increase in cytokine levels are known to be associated with RA $[1,2]$, the molecular basis of the disease remains unclear. Insight into the pathogenesis of RA - and hence effective treatment of RA - has been impeded by the heterogeneity of the disease. Not only can the disease course range from mild and self-limiting to severe and progressive, but also some patients respond well to early therapeutic intervention whereas others do not [3]. Therefore, there is a need for tests that can diagnose

\footnotetext{
* Correspondence: wrobins@stanford.edu

'Division of Immunology and Rheumatology, Department of Medicine,

Stanford University School of Medicine, Stanford, CA 94305, USA

Full list of author information is available at the end of the article
}

early-stage RA, as well as tests that can predict which RA patients will require and respond to anti-rheumatic therapies.

Diagnostic tests currently used in the management of early-stage RA are not sufficiently accurate, largely because they are based on detection of single biomarkers that are either not specific to RA, e.g. rheumatoid factor (RF) and C-reactive protein (CRP), or are present in only a subset of RA patients, e.g. autoantibodies that recognize cyclic citrullinated peptides $(\mathrm{CCP})$. Even when they correctly diagnose RA, current tests cannot adequately predict the course of the disease or the response to therapy because detection of a single biomarker cannot differentiate between the multiple, distinct subtypes of RA. Simultaneous analysis of multiple biomarkers may be more informative, yielding 'biomarker signatures' of RA subtypes. Indeed, we previously demonstrated that multiplex analysis of biomarkers in early-stage RA
C Biomed Central

() 2011 Chandra et al.; licensee BioMed Central Ltd. This is an open access article distributed under the terms of the Creative Commons Attribution License (http://creativecommons.org/licenses/by/2.0), which permits unrestricted use, distribution, and reproduction in any medium, provided the original work is properly cited 
could define molecular subtypes of RA that correlated with clinically identifiable RA subtypes [1,2]. Notably, the presence of autoantibodies targeting citrullinated proteins correlated with an increase in expression of proinflammatory cytokines [2]. In addition, we recently identified a biomarker signature of autoantibody specificities and cytokine levels that could distinguish between RA patients who will respond to anti-TNF treatment and those who will not [4].

Translation of these multiplex biomarkers onto a highly reproducible, automated platform is necessary for their use in robust validation studies and, ultimately, clinical practice. In this study, we developed such a highly reproducible, automated, multiplex biomarker assay and tested its performance in the diagnosis of RA and in the molecular stratification of RA patients into clinically relevant subtypes.

\section{Materials and methods}

\section{Roche multiplex automated assay}

Roche Professional Diagnostics (Roche Diagnostics $\mathrm{GmbH}$, Penzberg, Germany) is developing a multiplex platform called IMPACT (Immunological Multi-Parameter Chip Technology) that is based on a small polystyrene chip, as previously described [5]. During manufacturing, the chip is coated with a streptavidin layer onto which biotinylated markers - antibodies, proteins, or peptides - are spotted in vertical rows for the duplicate analysis of samples (Figure 1). Each chip contains up to 10 different markers, and each marker is arrayed on the chip as a vertical row of 10 to 12 spots; a minimum of five spots is required for determination of the level of a specific analyte in a sample. During the assay, the arrayed markers are probed with a small volume of sample and with a digoxigenylated secondary monoclonal antibody. The secondary antibody is then detected by the addition of an anti-digoxigenin antibody conjugated to a fluorescent latex label. This label enables sensitive detection of less than 10 individual binding events in a single spot, down to $\mathrm{fmol} / \mathrm{L}$ concentrations (Roche Diagnostics, Penzberg, Germany; proprietary data on file). After this final incubation with anti-digoxigenin antibody, chips are transferred to a detection unit where a charge-coupled device camera creates an image that is converted to signal intensities, and fluorescence intensity of the array features is quantified by image analysis. The IMPACT platform currently enables multiplex analysis of up to 10 analytes in a sandwich or indirect antibody assay format, requires only microliter quantities of serum samples, and is highly sensitive. The throughput of the prototype is 40 determinations per hour. One run is intended to comprise 100 single determinations, including standards and controls.
The chips and markers used in the present study are listed in Table 1; the sequences of the peptides spotted onto the chips are listed in Table S1 in Additional File 1. Autoantibody reactivities were measured in an indirect immunoassay in which candidate RA antigens were spotted onto the chips. Levels of analytes (e.g. inflammatory and bone-turnover markers) were measured in a sandwich immunoassay in which primary, capture antibodies were spotted onto the chips. All antigens and antibody pairs on these chronic inflammatory disease (CID) chips were developed by Roche Diagnostics. For measurement of RF, human anti-IgA and anti-IgM antibodies were spotted onto the chip as capture antibodies, and the RF they bound was then detected using biotinylated polymerized human IgG. Antigens on the synovial chips [see Table S1 in Additional File 1 were selected through screens performed in the laboratory of Robinson et al. [1] or our collaborators' laboratory [6]; they were then synthesized and spotted onto IMPACT chips by Roche Diagnostics. Using the appropriate chip-specific dilution buffers, we diluted the serum samples 1:10 for use in the synovial antigen 1 and 2, CID 3, and CID 4 chips, and 1:100 for use in the CID 1 chips. In the assays using the synovial antigen 1 and 2, CID 1, CID 3, or CID 4 chips, the arrayed antigens or antibodies were probed with $40 \mu \mathrm{l}$ of diluted serum sample, washed, and then probed with $40 \mu \mathrm{l}$ of digoxigenylated secondary monoclonal antibody. In assays using the chips containing markers of bone turnover (bone chips), the arrayed antibodies were probed with $40 \mu \mathrm{l}$ of serum at a 1:2 dilution and then $20 \mu \mathrm{l}$ of digoxigenylated monoclonal antibody. Standards specific to each type of chip were included in the assays using the CID 1, CID 3, CID 4, and bone chips, and levels of each analyte were calculated on the basis of the standard curves generated. Results for the synovial antigen 1 and 2 chips (for which standards have not yet been generated) were reported and analyzed as signal intensities. We minimized nonspecific binding by using fragments (Fab, Fab', or Fab'2) as capture antibodies and by using proprietary buffer reagents (in addition to the standard casein, BSA, and detergents) to minimize non-specific binding to the solid phase. For the indirect immunoassays (CCP and synovial chips), a proprietary detection antibody was used that has been optimized to ensure minimal nonspecific binding. Extensive evaluation revealed that diluting the sample does not significantly influence non-specific binding (data not shown).

\section{Multiplex cytokine assay}

To measure cytokine or chemokine levels in sera, we used the Milliplex Map Human cytokine/chemokine kit (Millipore, Billerica, MA, USA) run on the Luminex 200 platform coupled with BioRad Bio-Plex software 


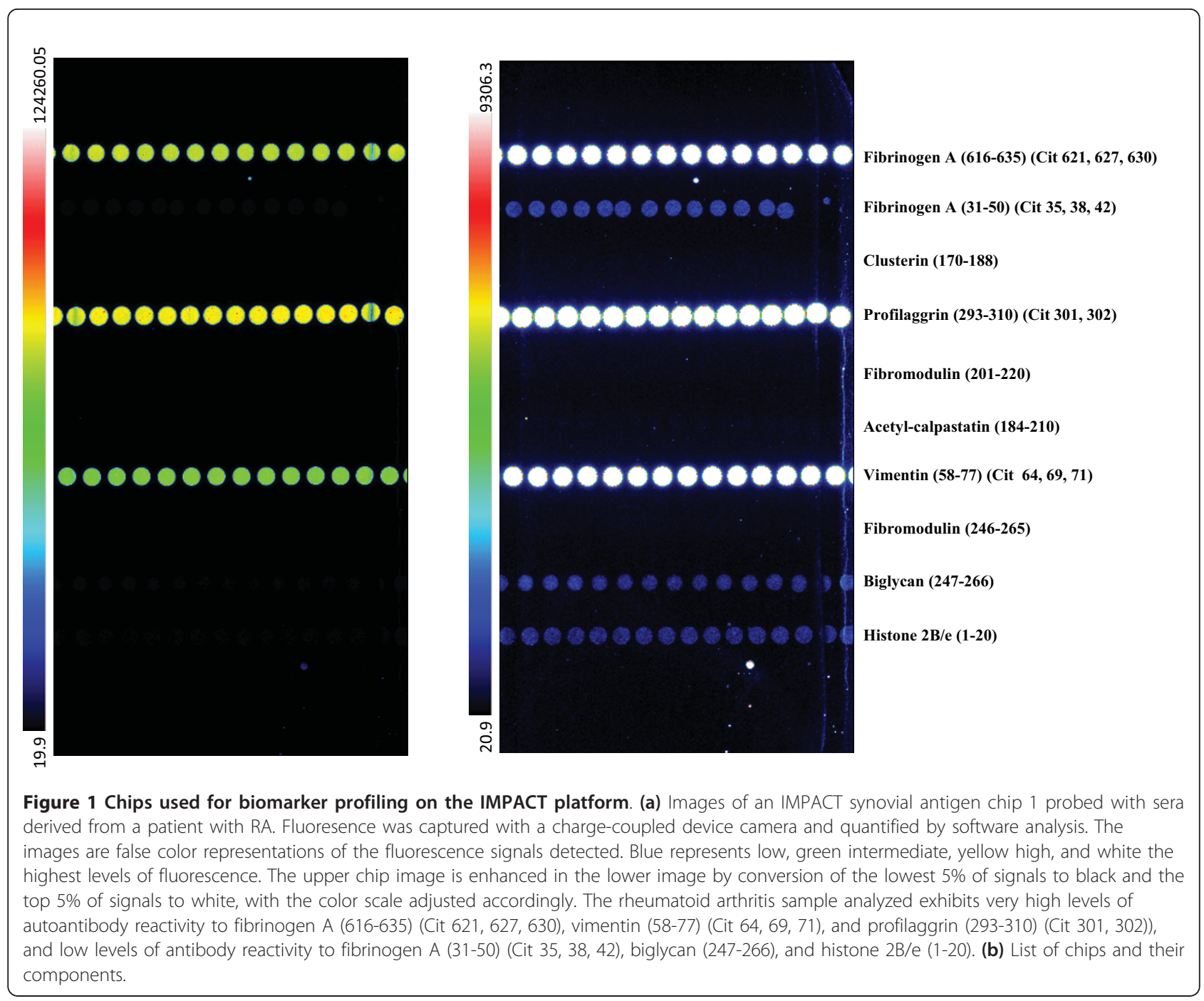

(BioRad, Hercules, CA, USA), according to the manufacturers' protocols. The cytokines and chemokines measured were eotaxin, fibroblast growth factor 2, granulocyte macrophage colony-stimulating factor, IL$1 \alpha$, IL-1 $\beta$, IL-6, IL-12 (p40), IL-12 (p70), IL-15, IL-17, IP-10, monocyte chemoattractant protein 1 (MCP-1), and TNF. To prevent RF from bridging capture and detection antibodies in the immunoassays, we added Heteroblock (Omega Biologicals, Bozeman, MT, USA) to the sera at a final concentration of $3 \mu \mathrm{g} / \mathrm{ml}$ (we have shown that this concentration of Heteroblock eliminates false augmentation of the readout by heterophilic antibodies [2]). Calibration controls and recombinant standards were used as specified by the manufacturer.

\section{Single automated assays}

Roche Tina-Quant assays run on a fully automated platform (Roche/Hitachi COBRAS C system) were used for the individual, automated measurement of CRP and RF levels in patient sera. In the CRP assay, latex particles coated with monoclonal anti-CRP antibodies agglutinate with human CRP. In the RF assay, latex-bound, heatinactivated IgG reacts with RF to form antigen-antibody complexes. Both assays use turbidimetry to determine latex agglutination, which occurs in cases of positive test results.

\section{Serum samples}

All patient serum samples were used after obtaining informed consent from the patients and under human subjects protocols approved by the Stanford University Institutional Review Board. Samples from RA patients were obtained from ARAMIS (Arthritis, Rheumatism and Aging Medical Information System), which includes a biobank of serum samples from 793 Caucasian RA patients who were recruited by a consortium of 161 practising rheumatologists throughout the USA $[1,2,7,8]$. All patients met the 1987 Arthritis College of 
Table 1 Chips and markers used on the IMPACT platform*

\begin{tabular}{|c|c|c|}
\hline \multirow[t]{2}{*}{ Chip name } & \multicolumn{2}{|c|}{ Chip components } \\
\hline & Antigens & Capture antibodies \\
\hline \multirow[t]{10}{*}{ Synovial antigen chip 1} & Histone 2B/e (1-20) & \\
\hline & Biglycan (247-266) & \\
\hline & Fibromodulin (246-265) & \\
\hline & Vimentin (58-77) (Cit 64, 69, 71) & \\
\hline & Acetyl-calpastatin (184-210) & \\
\hline & Fibromodulin (201-220) & \\
\hline & Profilaggrin (293-310) (Cit 301, 302) & \\
\hline & Clusterin (170-188) & \\
\hline & Fibrinogen A (31-50) (Cit 35, 38, 42) & \\
\hline & Fibrinogen A (616-635) (Cit 621, 627, 630) & \\
\hline \multirow[t]{8}{*}{ Synovial antigen chip 2} & Histone 2A (95-114) & \\
\hline & Profilaggrin (293-310) (Cit 301, 305) & \\
\hline & HSP60 (287-297) & \\
\hline & Serine protease 11 (433-452) & \\
\hline & Osteoglycin (177-196) & \\
\hline & Apolipoprotein E (277-296) (Cit 278, 292) & \\
\hline & Clusterin (334-353) (Cit 336, 339) & \\
\hline & COMP (453-472) & \\
\hline \multirow[t]{3}{*}{ CID 1} & & anti-CRP \\
\hline & & anti-IgA (for RF measurement) \\
\hline & & anti-IgM (for RF measurement) \\
\hline \multirow[t]{4}{*}{ CID 3 chip 1} & Cit peptide 1 & \\
\hline & Cit peptide 2 & \\
\hline & Cit peptide 3 & \\
\hline & Cit peptide 4 & \\
\hline \multirow[t]{7}{*}{ CID 3 chip 2} & Cit peptide 5 & \\
\hline & Cit peptide 6 & \\
\hline & Cit peptide 7 & \\
\hline & Cit peptide 8 & \\
\hline & Cit peptide 9 & \\
\hline & Cit peptide 10 & \\
\hline & Cit peptide 11 & \\
\hline \multirow[t]{5}{*}{ CID 4} & & anti-MMP 3 \\
\hline & & anti-IL-6 \\
\hline & & anti-S100 protein A8/A9 \\
\hline & & anti-E-Selectin \\
\hline & & anti-HABP \\
\hline \multirow[t]{4}{*}{ Bone } & & anti-PTH \\
\hline & & anti- $\beta$ Crosslaps \\
\hline & & anti-Osteocalcin \\
\hline & & anti-P1NP \\
\hline
\end{tabular}

${ }^{*}$ Candidate rheumatoid arthritis antigens were spotted on the chip for measurement of autoantibody reactivities. Primary antibodies were spotted on the chip for measurement of analyte (e.g. inflammatory mediators and products of bone turnover) levels.

Cit, citrullinated; HSP 60, heat shock protein 60; COMP, cartilage oligomeric matrix protein; CRP, C-reactive protein; MMP3, matrix metalloproteinase 3; IL-6, interleukin-6; HABP, hyaluronic acid binding protein; PTH, parathyroid hormone; P1NP, procollagen type 1 amino-terminal propeptide.

Rheumatology criteria [9] and had RA of less than six months' duration. We used a randomisation algorithm to select serum samples from 120 patients in the ARAMIS cohort. The baseline characteristics of this subgroup of patients with early RA were assessed and found to be comparable with those of the whole cohort of patients [7]. Psoriatic arthritis (PsA) samples were provided by James T. Elder and represent a mixture of different subtypes of PsA (25\% RA-like, 25\% mutilans, and $50 \%$ distal interphalangeal predominant disease). 
Ankylosing spondylitis (AS) samples were provided by John Reveille and represent a cohort of patients with active axial and/or uveal disease. Serum samples from healthy individuals were obtained from Bioreclamation, Inc (Hicksville, NY, USA). All serum samples were shipped on dry ice, stored at $-80^{\circ} \mathrm{C}$, and subjected to one freeze-thaw cycle before being analyzed.

In assessing the analytical precision of the IMPACT assay, we used serum samples from the REFLEX study, a phase III trial on the efficacy of rituximab on a background of methotrexate in RA refractory to anti-TNF therapy [10]. We used only samples obtained at baseline.

\section{Statistical analysis}

Values for each marker were divided by six times the mean value obtained for that marker in the healthy control samples and then log transformed. These normalized values were analyzed by SAM (Significance Analysis of Microarrays) [11,12]. Output was sorted based on false discovery rates (FDRs) in order to identify antigens with the greatest differences in autoantibody reactivity, or cytokines with the greatest differences in concentrations, between patients with RA, patients with other inflammatory arthritides, and healthy individuals. Most of our comparisons involved high-dimensional data, and we therefore used FDR for our exploratory analyses, an analytical method that obviates the need for multiple corrections when using high-dimensional data [11]. We then used hierarchical clustering software (Cluster ${ }^{\circledR}$ 3.0, developed by Michael Eisen at Stanford University, Stanford, California) to arrange the SAM results according to similarities among patient samples in autoantibody specificities or cytokine levels, and Java Treeview ${ }^{\circledR}$ (Java Treeview 1.1.3, developed by Alok J. Saldanha at Stanford University, Stanford, California) to graphically display the results.

To evaluate the IMPACT assay's diagnostic sensitivity and specificity, we used a subpanel of markers from the original array results - markers identified by univariate analysis as ones that differentiate between patients with RA and patients with other arthritides. A fluorescent value three times the mean value of that obtained in healthy control samples was defined as positive because this cutoff yielded greater specificity than a cutoff of three standard deviations above the mean. Similarly, because we had fewer healthy controls than RA cases, this method provided greater specificity than did Z-normalization. We excluded RF values from the analysis when comparing RF-positive and RFnegative subgroups, and CCP values when comparing antiCCP-positive and anti-CCP-negative subgroups.

\section{Results}

\section{Analytical precision of IMPACT assays}

To develop a system for the multiplex analysis of different types of biomarkers in the sera of RA patients, we used a bead-based commercial assay (Millipore/Luminex) to evaluate cytokine levels, and an array-based assay in development (IMPACT) to evaluate autoantibody reactivities and bone turnover. To determine the intra-assay reproducibility achieved with the IMPACT platform, we performed 21 replicate measurements of each of nine markers within one run on the IMPACT platform. The intra-assay coefficients of variance (CV) ranged from 1.5 to $9.0 \%$ (Figure 2a). To determine inter-assay reproducibility, we compared measurements obtained from 5 to 15 independent runs of the same sample at low, medium, and high dilutions; this was done for eight of the markers present on the IMPACT platform. Analysis demonstrated inter-assay CVs ranging from 1.1 to $14.9 \%$ (Figure 2a). Notably, these results compare favorably with CVs obtained with current commercial ELISA tests for RF (which yield intra-assay CVs of $6 \%$ and inter-assay CVs of 8\%) [13] and CCP (which yield intra-assay CVs of 4.8 to $13 \%$ and inter-assay CVs of 9 to $17 \%$ ) [14].

To assess the correlation between IMPACT multiplex assays and single automated assays, we used both the IMPACT and the Roche/Hitachi cobas c platforms to measure RF and CRP in baseline serum samples from subjects enrolled in the REFLEX study [10]. Linear regression analysis demonstrated that the correlation between the results from the multiplex assay and those from the single assay was good, with correlation coefficients of 0.92 for RF and 0.97 for CRP (Figures $2 b$ and 2c). Analysis of the bone-turnover markers with IMPACT was previously described, the results of which correlated well with those of corresponding single automated assays [5].

\section{Biomarker signatures define distinct arthritides and arthritis subtypes}

To identify molecular signatures of arthritis subtypes, we used antigen-containing chips on the IMPACT platform to measure autoantibody reactivities and bone-turnover markers [5], and bead-based assays on the Luminex platform to measure cytokines, in serum samples from 120 patients with RA, 27 patients with AS, 28 patients with PsA, and 25 healthy individuals. Values were normalized as described in the methods, subjected to hierarchical clustering, and displayed as a softwaregenerated heat map (Figure 3). As expected, autoantibody levels were significantly higher in RA patients than in AS patients, PsA patients, or healthy controls. However, within the pool of RA patients were subgroups with distinct patterns of autoantibody specificities, including a subgroup with minimal autoantibody reactivity. Elevations in cytokine levels clearly distinguished certain subsets of patients with RA, AS, or PsA from healthy individuals. Certain subsets of arthritis patients 


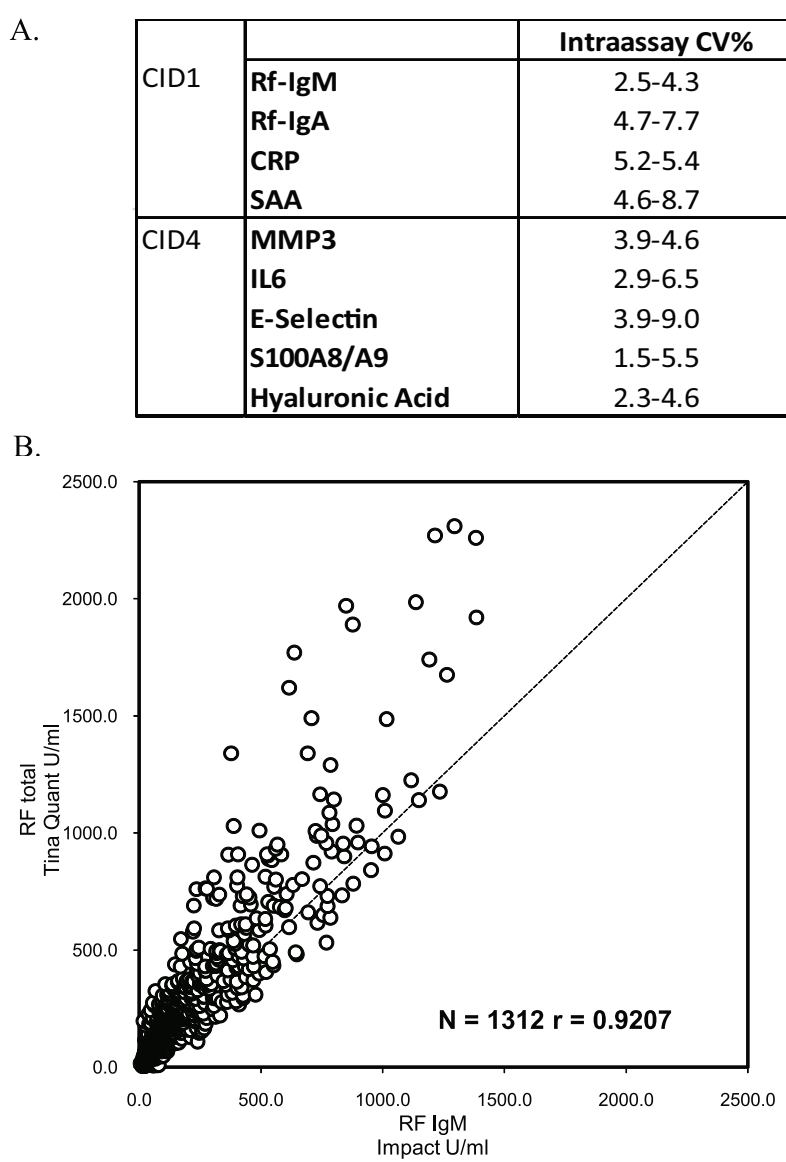

\begin{tabular}{|c|}
\hline Interassay CV\% \\
\hline $1.1-4.0$ \\
$1.9-6.3$ \\
$5.3-12.6$ \\
$2.7-4.8$ \\
\hline $8.6-14.6$ \\
$11.6-12.0$ \\
$7.2-8.8$ \\
$5.0-14.9$ \\
n.t. \\
\hline
\end{tabular}

B.

C.

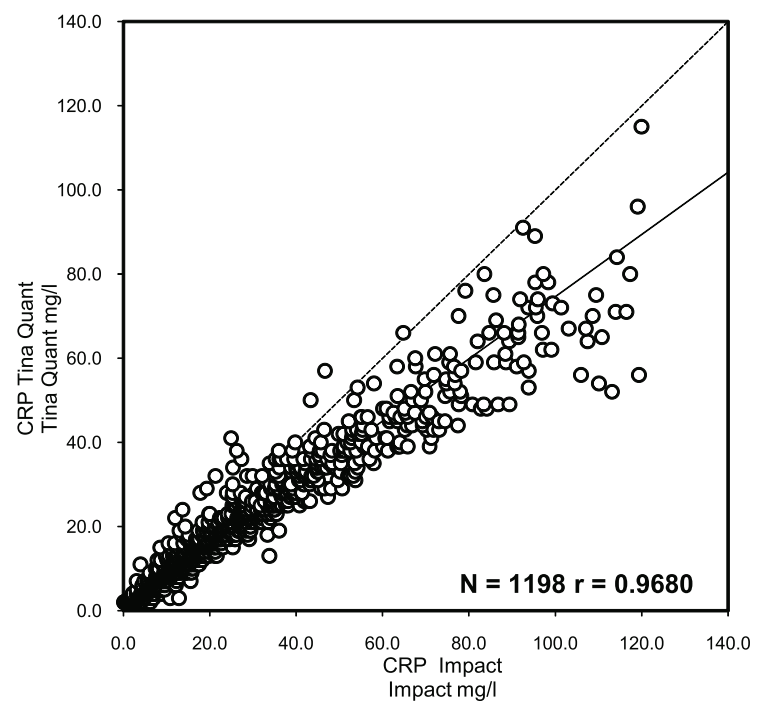

Figure 2 Analytical precision of selected IMPACT assays and comparison with standard single assays. (a) Analytical precision. Intra-assay coefficients of variance (CV) were generated by performing 21 replicate measurements of each of nine markers in one sample within one run on the IMPACT platform. Inter-assay CVs were calculated based on results from 5 to 15 independent runs of the same sample on the IMPACT platform. The range of the CV for each marker corresponds to that of three independent pools of sample analyzed at low, medium, and high concentrations. (b) Correlation of values obtained with the Roche IMPACT platform with those obtained with the standard Roche Tina Quant (latex aggregation) assay. IgM autoantibody reactivity to rheumatoid factor (IgM-RF) in 1,312 RA serum samples was measured with the IMPACT platform and with Tina Quant assay. C-reactive protein (CRP) levels in 1,198 RA serum samples were measured with the IMPACT platform and with Tina Quant assay. Linear regression was used to determine the correlation between the multiplex chip assay (IMPACT) and the standard single assay (Tina Quant). IL-6, interleukin-6; MMP3, matrix metalloproteinase 3; SAA, serum amyloid A.

had lower cytokine levels than did other patients with the same diagnosis. As autoantibody production is not typically a feature of PsA, the detection of autoantibodies in several patients diagnosed with PsA (Figure 3) raises the possibility that evaluation of a larger panel of autoantibodies than that measured by the commercially available assays may be able to correct misdiagnosis.

In contrast to previous findings $[15,16]$ we did not find an association between RA and markers of bone turnover. This is perhaps not surprising given that our analysis was done using a cohort of patients with earlystage RA, and erosion of bone occurs in established and advanced RA. In contrast, an association between AS and elevated levels of markers of bone turnover - specifically, beta crosslaps, and osteocalcin - was revealed in the course of the biomarker analysis (Figure 4), suggesting that activation of bone-turnover pathways, exceeding that seen in RA or PsA, occurs in AS. Also intriguing was the increase in levels of the bone-marker parathyroid hormone. However, because levels of parathyroid hormone are heavily influenced by vitamin $\mathrm{D}$ status [17] (a variable not accounted for in our study), firm conclusions about associations between parathyroid hormone and AS cannot be drawn from our present data. Levels of proinflammatory cytokines were also significantly higher in AS patients than in healthy individuals, in line with previous findings $[18,19]$.

\section{Association of biomarker signatures with parameters predictive of severe RA}

Using research-grade platforms, we previously demonstrated an association between specific biomarker 


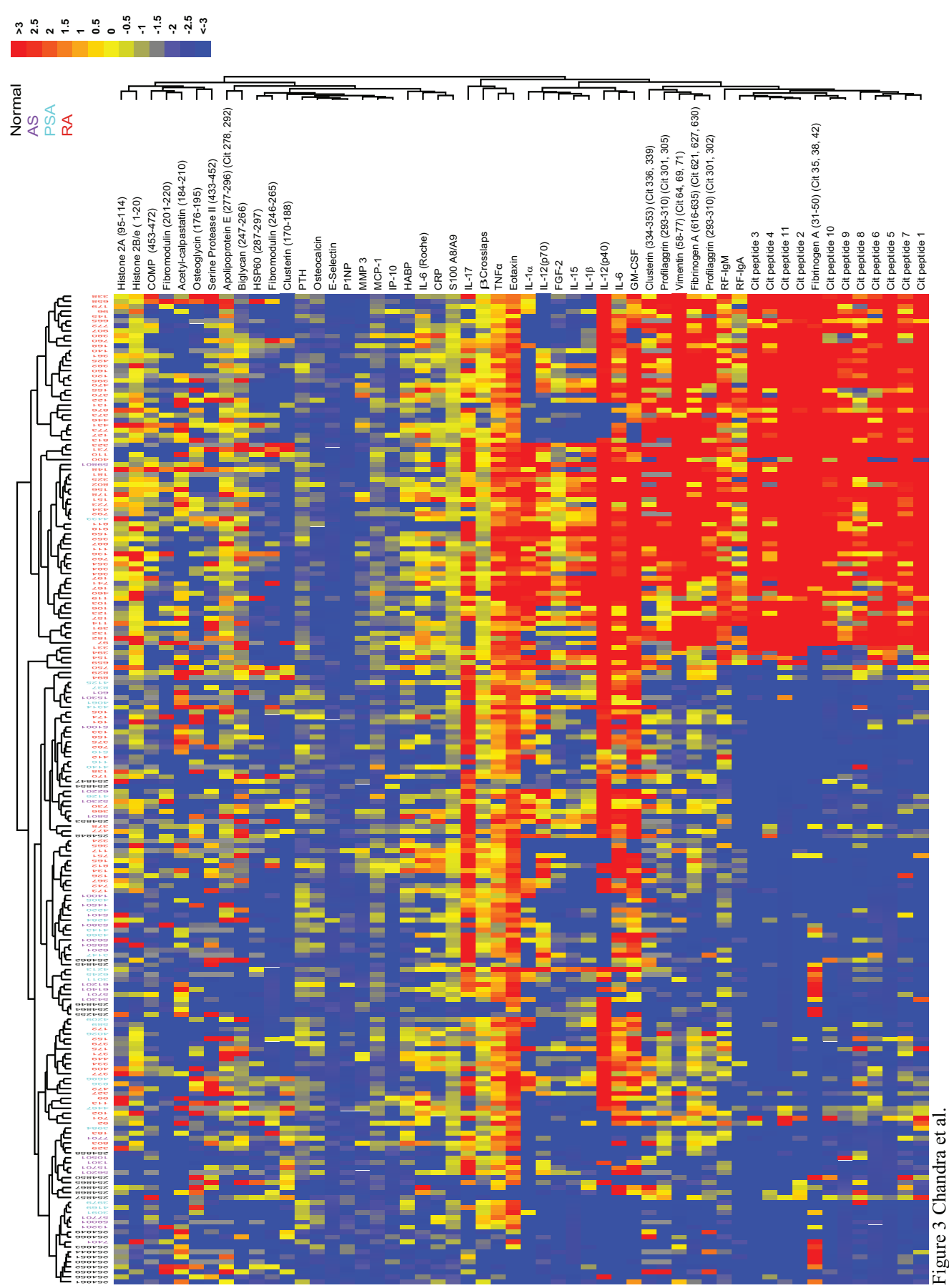

Figure 3 Proteomic characterization of serum samples from patients with rheumatoid arthritis, psoriatic arthritis, or ankylosing spondylitits. Autoantibody reactivities and levels of bone-turnover products in serum samples from 120 patients with rheumatoid arthritis (RA), 27 patients with ankylosing spondylitits (AS), 28 patients with psoriatic arthritis (PSA), and 25 healthy individuals were measured on the IMPACT platform. Cytokine levels were measured with a bead-based assay (Millipore) run on the Luminex platform. Values were normalized as described in the methods and subjected to hierarchical clustering; individual patients are listed above the heat map and the individual cytokines and antigens are listed to the right of the heat map. Cytokine levels and autoantibody reactivities are displayed, with blue representing a decrease relative to the mean value obtained in samples from healthy individuals, yellow no change, and red an increase. Cit, citrullinated; COMP, cartilage oligomeric matrix protein; CRP, C-reactive protein; FGF-2, fibroblast growth factor 2; GM-CSF, granulocyte macrophage colonystimulating factor; HABP, hyaluronic acid binding protein; HSP 60, heat shock protein 60; IL, interleukin; MCP-1, monocyte chemoattractant protein 1; MMP3, matrix metalloproteinase 3; P1NP, procollagen type 1 amino-terminal propeptide; PTH, parathyroid hormone; RF, rheumatoid factor; $T N F \alpha$, tumor necrosis factor $\alpha$. 


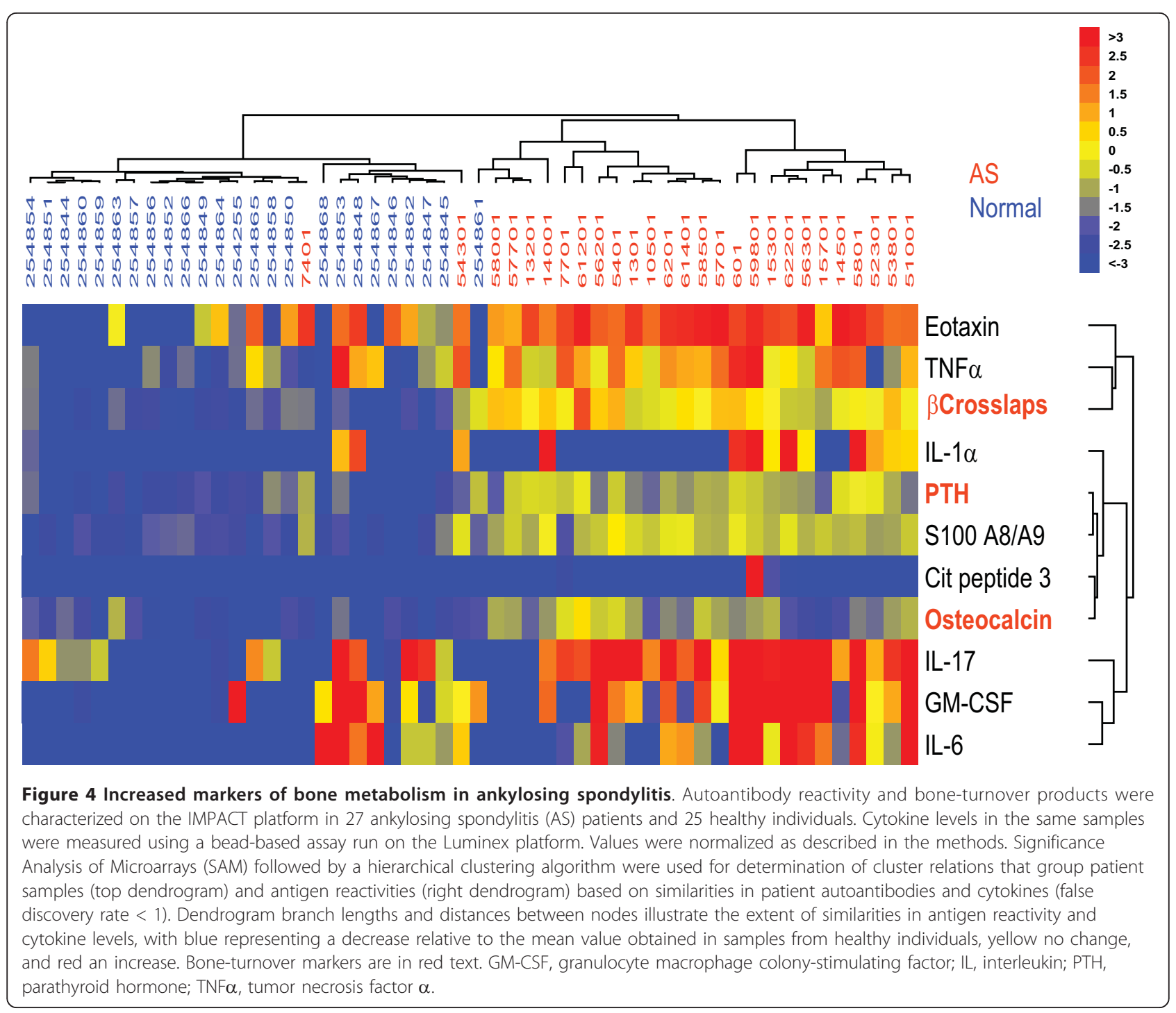

signatures and the presence of RF, anti-CCP antibodies, or shared-epitope (SE) alleles [1,2], each of which predicts progression to severe RA [20]. To determine whether the automated IMPACT platform could recapitulate this finding, we used the IMPACT platform in conjunction with bead-based multiplex assays to characterize serum samples from 120 RA patients, of which 73 had anti-CCP antibodies (as assessed by the IMPACT assay), 78 had RF (as assessed by the IMPACT assay), and 74 had one or two SE alleles. We performed our analysis using a subset of the antigen markers we used previously $[1,2,4]$, as well as an additional set of analyte assays previously developed for use on the IMPACT platform (Figure 1). Data from the CCP-containing chips used to determine anti-CCP-antibody status of the patient samples (i.e., CID 3 chips 1 and 2) were excluded from analyses comparing patients on the basis of presence or absence of anti-CCP antibodies.
We again demonstrate a clear association between the presence of anti-CCP (Figure 5) or RF (Figure 6) antibodies and increased targeting of RA-associated autoantigens - most citrullinated, but some native. Notably, distinct but overlapping sets of antigens were targeted in RF-positive patients compared with anti-CCP-antibody-positive patients. Likewise, the pattern of increases in cytokine levels showed both differences and similarities between RF-positive patients and anti-CCP-antibody-positive patients. Despite the strong association between seropositivity (the presence of RF and/or antiCCP antibodies) and elevation of serum cytokines, a subset of seronegative patients had significantly elevated serum cytokines, possibly reflecting a subpopulation more clinically and immunologically similar to those who can be defined as seropositive. When we sought to identify differences on the basis of the presence or absence of SE alleles, we found that the presence of SE 


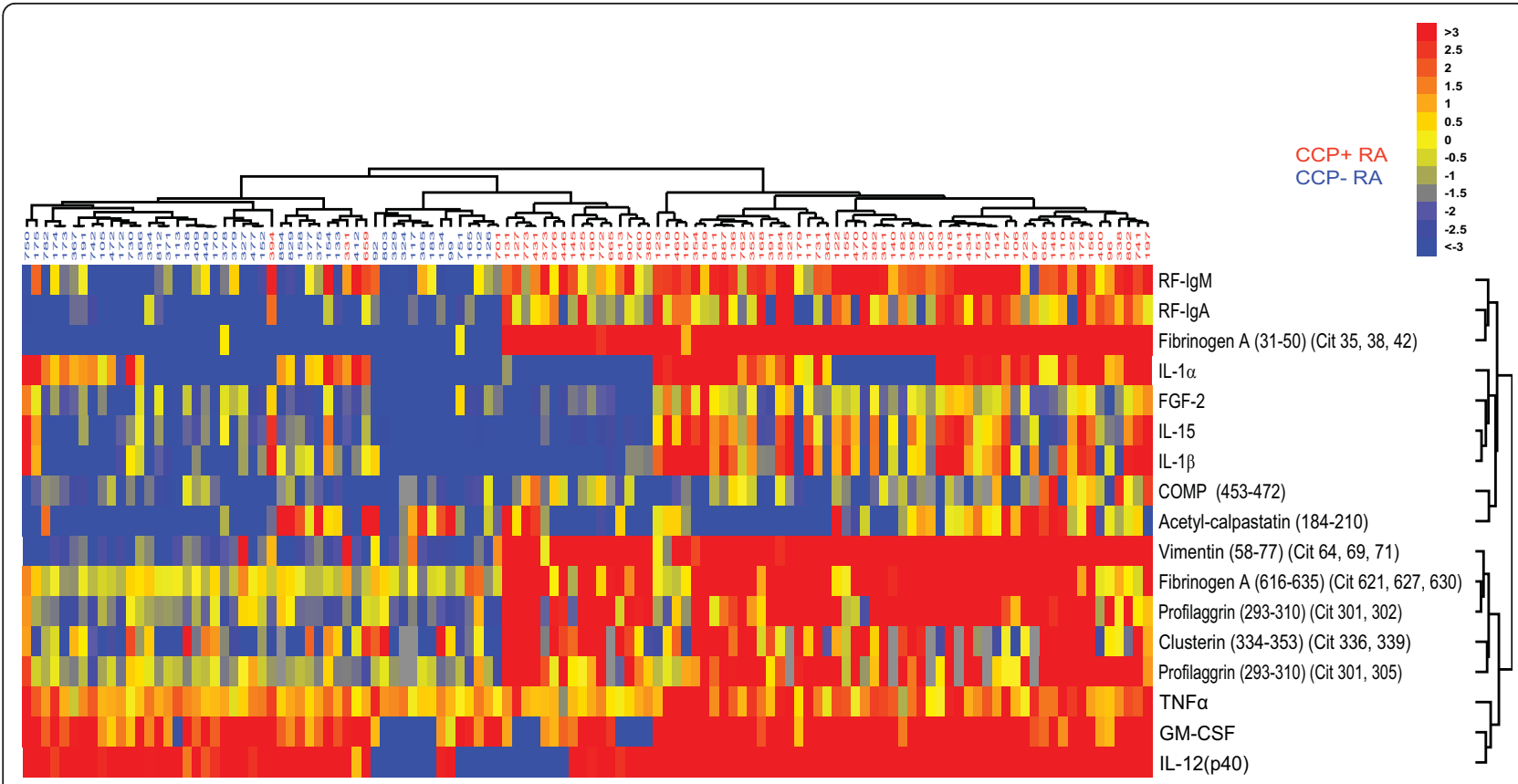

Figure 5 Autoantibodies and cytokine levels stratified according to anti-CCP seropositivity. Autoantibody and cytokine levels are higher in anti- cyclic citrullinated peptide (CCP)-antibody-positive than in anti-CCP-antibody-negative RA. Serum samples from 73 patients with antiCCP-antibody-positive RA and from 47 patients with anti-CCP-antibody-negative RA were analyzed. Chips containing CCP were excluded from this analysis. Autoantibody reactivity was assessed on the IMPACT platform and cytokine levels were measured in a bead-based assay run on the Luminex platform. For assays run on the IMPACT platform, values were normalized as described in the methods. Significance Analysis of Microarrays (SAM) followed by a hierarchical clustering algorithm were used to determine cluster relations that group patient samples (top dendrogram) and antigen reactivities (right dendrogram) on the basis of similarities in patient autoantibody and cytokine profiles (false discovery rate $<1$ ). Dendrogram branch lengths and distances between nodes illustrate the extent of similarities in antigen reactivity and cytokine levels, with blue representing a decrease relative to the mean value obtained in samples from healthy individuals, yellow no change, and red an increase. Cit, citrullinated; COMP, cartilage oligomeric matrix protein; FGF-2, fibroblast growth factor 2; GM-CSF, granulocyte macrophage colonystimulating factor; IL, interleukin; RF, rheumatoid factor; $T N F \alpha$, tumor necrosis factor $\alpha$.

alleles was associated with increased targeting of RAassociated autoantigens; however, unlike the presence of RF or anti-CCP antibodies, the presence of SE alleles alone was not associated with elevations in serum cytokines (Figure 7). There was no significant difference between carrying one versus two copies of the SE allele (data not shown).

\section{Autoantibody and cytokine signatures as sensitive and specific diagnostics of RA}

Using univariate analysis, we determined which of the biomarkers (out of 31 autoantigens, 4 bone markers, 5 inflammatory mediators, and 14 cytokines) distinguish RA patients from a pool of 120 patients with early-stage RA, 27 patients with AS, 28 patients with PSA, and 25 healthy individuals. We found that a panel of six autoantigens distinguished RA. We then used the same serum samples to evaluate the diagnostic sensitivity and specificity of different combinations of the individual autoantigens in this differentiating panel of six biomarkers. The sensitivity and specificity of these subpanels in the differential diagnosis of RA were similar to that of anti-CCP status [21] and better than that of RF status [22] (Table 2).

\section{Discussion}

We report the development of a highly reproducible, automated, multiplex biomarker assay that can reliably distinguish RA patients from healthy individuals or patients with other inflammatory arthritides. Multiplex measurement of a subset of the differentiating biomarkers provided high sensitivity and specificity in the diagnostic discrimination of RA. Furthermore, the biomarker profiles we identified enabled stratification of RA patients into distinct, clinically relevant subtypes.

Current clinical tests fall short of being accurate and all-encompassing diagnostics of RA because RF is not specific to RA and anti-CCP antibodies are not produced in all cases of RA. Compared with single-biomarker detection, multiplex-biomarker detection - by casting the net wider - provides greater sensitivity and specificity of diagnosis. Although they remain to be 


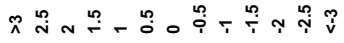

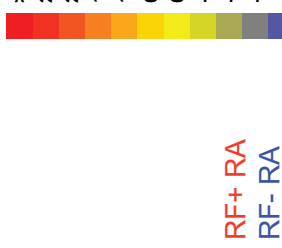

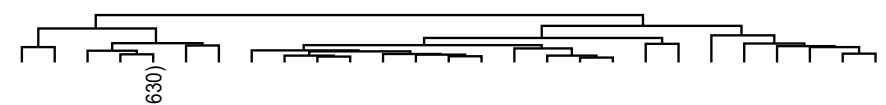

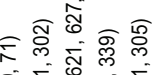

iि

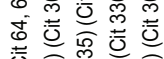

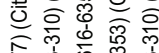

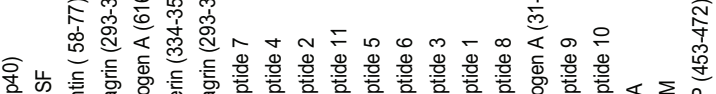

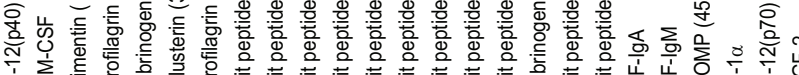

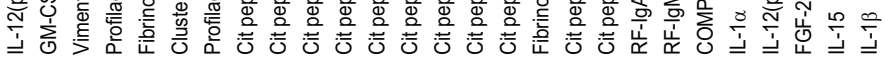

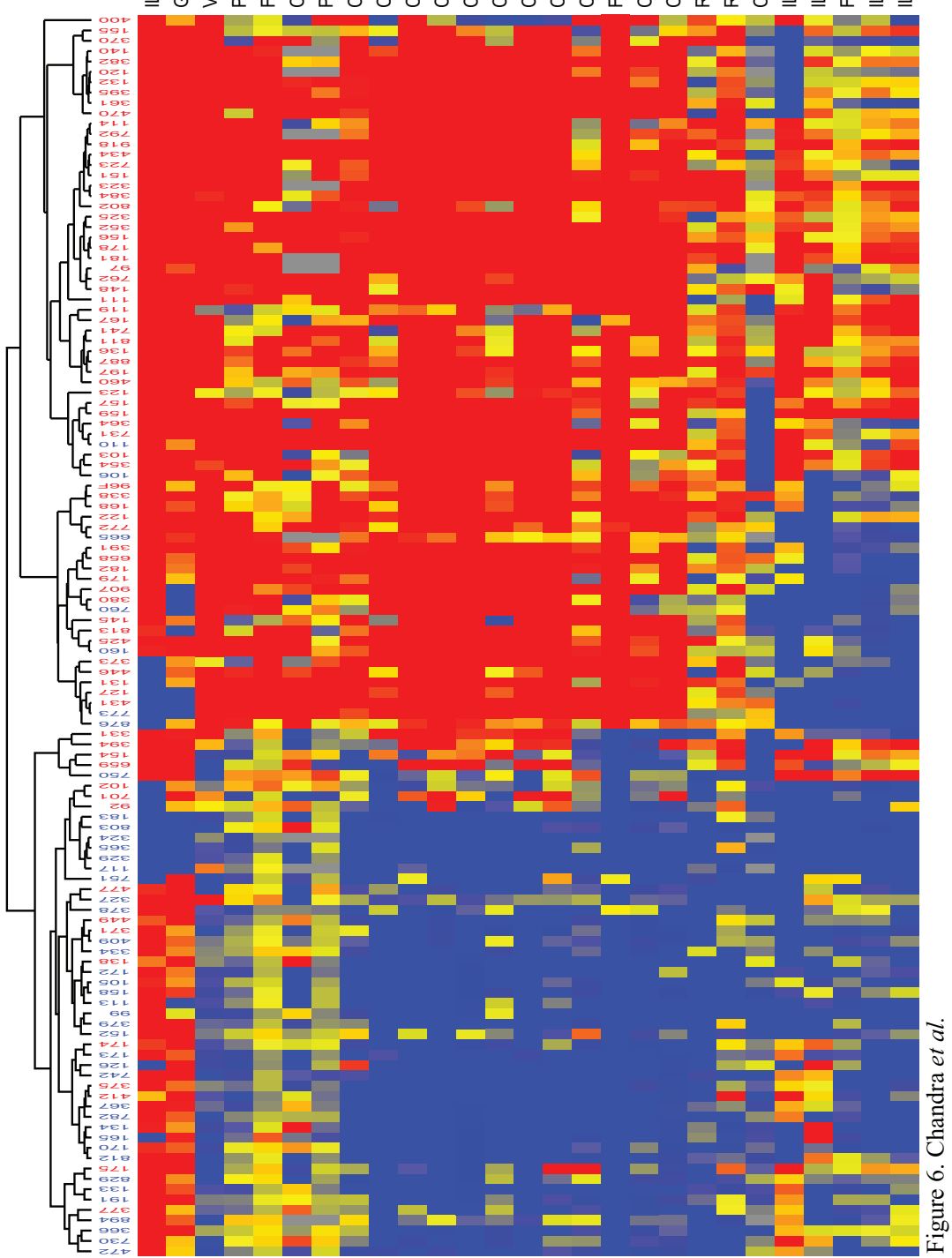

Figure 6 Autoantibodies and cytokine levels stratified according to RF seropositivity. Autoantibody and cytokine levels are higher in rheumatoid factor (RF)-positive RA than in RF-negative RA. Serum samples from 78 patients with RF-positive RA and from 42 patients with RFnegative RA were analyzed. Autoantibody reactivity was assessed on the IMPACT platform and cytokine levels were measured in a bead-based assay run on the Luminex platform. For assays run on the IMPACT platform, values were normalized as described in the methods. Significance Analysis of Microarrays (SAM) followed by a hierarchical clustering algorithm were used to determine cluster relations that group patient samples (top dendrogram) and antigen reactivities (right dendrogram) on the basis of similarities in patient autoantibody and cytokine profiles (false discovery rate $<1$ ). Dendrogram branch lengths and distances between nodes illustrate the extent of similarities in antigen reactivity and cytokine levels, with blue representing a decrease relative to the mean value obtained in samples from healthy individuals, yellow no change, and red an increase. Cit, citrullinated; COMP, cartilage oligomeric matrix protein; FGF-2, fibroblast growth factor 2; GM-CSF, granulocyte macrophage colony-stimulating factor; IL, interleukin; RF, rheumatoid factor. 


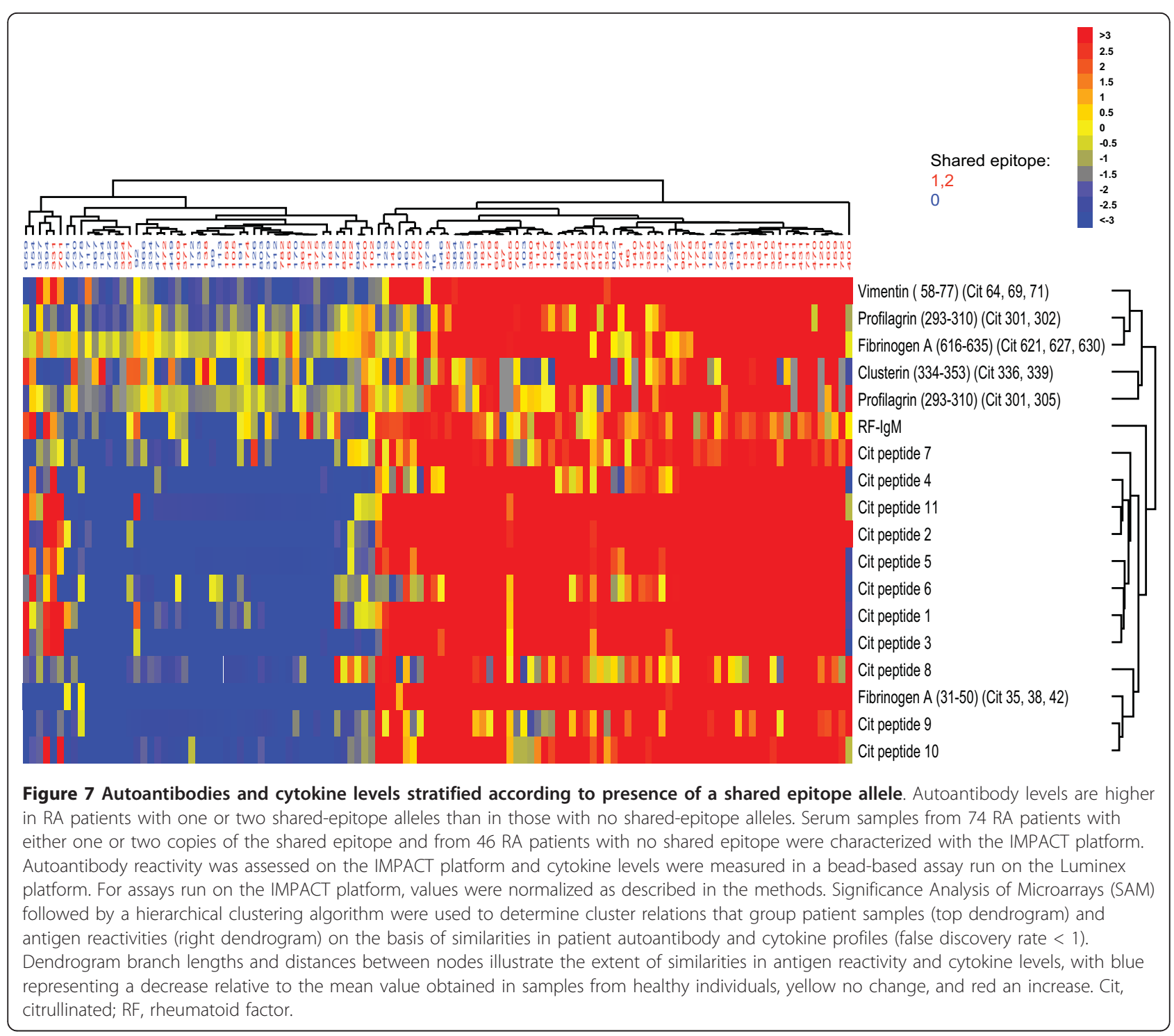

validated in independent cohorts of RA patients, our preliminary results suggest that our biomarker assay has the potential to provide greater diagnostic sensitivity and specificity than that provided by current clinical tests. Including in our analysis a larger number of control patients with non-RA inflammatory diseases should allow us to further increase the sensitivity and specificity of our biomarker assay. Whereas the commercial antiCCP-antibody assay relies on the measurement of antibody reactivity against a mixture of different citrullinated peptides, our multiplex biomarker assay allows measurement of antibody reactivity against each of several different citrullinated peptides independently, thus enabling more-precise diagnostic characterization. Moreover, the integrated evaluation of multiple additional biomarkers (i.e. autoantibody specificities, cytokine levels, and bone-turnover products) enables the stratification of RA into disease subtypes and provides further insight into disease pathogenesis at the individual level. For instance, our biomarker assay identified a subset of seronegative RA patients who had elevations in serum cytokines suggestive of more aggressive disease, an association that would have gone undetected with current clinical tests.

As RA is such a heterogeneous disease, diagnosis must be accompanied by prognosis in order to identify which patients with early-stage RA are in need of aggressive therapeutic intervention. The presence of serum RF or anti-CCP antibodies is associated with progression to severe RA [23-25]. When combined, these two biomarkers offer a somewhat improved prognostic capability [26]. Although we did not observe an association of bone-turnover markers with early-stage RA in this study, elevations in markers of bone and cartilage 
Table 2 Performance characteristics of multiplex-assayed autoantibody profiles in the diagnosis of rheumatoid arthritis

\begin{tabular}{lcccc}
\hline $\begin{array}{l}\text { Number of positive } \\
\text { biomarkers* }\end{array}$ & PPV & NPV & Sensitivity & Specificity \\
\hline 1+ markers & $79.5 \%$ & $92.6 \%$ & $96.7 \%$ & $62.5 \%$ \\
2+ markers & $91.8 \%$ & $89.7 \%$ & $93.3 \%$ & $87.5 \%$ \\
3+ markers & $95.3 \%$ & $79.8 \%$ & $84.2 \%$ & $93.8 \%$ \\
4+ markers & $95.9 \%$ & $61.1 \%$ & $59.2 \%$ & $96.3 \%$ \\
\hline
\end{tabular}

*Biomarker panel: histone 2B/e (1-20), vimentin (58-77) (Cit 64, 69, 71), fibrinogenA (616-635) (Cit 621, 627, 630), COMP (453-472), profilaggrin (293310) (Cit 301, 305), RF-IgA. The cut-off value for positivity was defined as three times the mean value obtained in samples from healthy individuals. PPV, positive predictive value; NPV, negative predictive value.

turnover [27] also have been proposed to predict a more destructive course of RA, as have elevations in acutephase reactants [28]. Multiplex biomarker detection should be more accurate and informative than singlebiomarker detection in RA prognosis, as it is in diagnosis. We show here that multiplex biomarker detection in early-stage RA can identify biomarker signatures that are associated with immunological (presence of anti$\mathrm{CCP}$-antibodies or RF) and genetic (possession of SE alleles) parameters predictive of more severe RA. Unfortunately, information on the degree of radiographic joint damage at the time of diagnosis, a powerful predictor of disease outcome, was not available for the cohort we analyzed. In addition, the greater use of disease-modifying anti-rheumatic drugs in patients with RF-positive RA confounded attempts to correlate our biomarker signatures with disability at diagnosis (as assessed by the Stanford Health Assessment Questionaire), a good predictor of later functional impairment [29]. In addition to validating out present findings in an independent cohort of patients, we aim to evaluate the prognostic utility of our assay. Given that our biomarker panel enables disease stratification and yields detailed molecular information, we expect that it will provide more precise prognosis than that achieved in the clinic at present.

Although not an overt objective of the present study, our biomarker analysis revealed that AS is associated with elevated levels of bone-turnover markers and cytokines, in line with previous findings $[18,30]$. The small number of AS patients included in this study precludes any firm conclusions, but this observation suggests that our multiplex platform may be useful in developing a diagnostic or prognostic test for AS - a major unmet clinical need.

This exploratory study has several limitations. Given that we were unable to adjust for treatment-related effects on the studied biomarkers, it is possible that use of immunosuppressant therapy could affect levels of serum cytokines and thereby confound interpretation of our data. In addition, the ARAMIS RA cohort studied represents a Caucasian, American, early-RA cohort, and therefore it is possible that our findings cannot be extrapolated to all RA patients; our findings remain to be validated in independent and more diverse cohorts. In addition, the fact that RA and control patients were not matched by demographics or by handling of their serum samples could bias our results.

\section{Conclusions}

In the diagnosis and prognosis of RA, measurement of a single biomarker is not sufficiently sensitive or accurate, and individual measurement of multiple biomarkers is labor intensive and therefore expensive. Automated multiplex biomarker analyses can help to reduce the laboratory workload involved in the analysis of multiple biomarkers and can provide greater sensitivity and specificity. However, their use in clinical trials has been hampered by their limited reproducibility between and within multiplex platforms. The multiplex system we developed in this study is ideally suited to the simultaneous analysis of multiple biomarkers because it uses a standardized assay platform and is highly automated, allowing high-throughput reproducibility across clinical laboratories. Here we demonstrate the effectiveness of this multiplex biomarker assay in stratifying RA into clinically relevant subtypes. The ability to classify RA patients in an automated and reproducible manner paves the way for further studies aimed at attaining personalized medicine for RA.

\section{Additional material}

Additional file 1: Sequences of peptides spotted on synovial

antigen chip 1 and 2 . Supplementary table showing the amino acid

sequences of the peptides spotted onto synovial antigen chips 1 and 2 .

\section{Abbreviations}

AS: ankylosing spondylitis; CCP: cyclic citrullinated peptide; CID: chronic inflammatory disease; COMP: cartilage oligomeric matrix protein; CRP: Creactive protein; $\mathrm{CV}$ : coefficient of variance; FDR: false discovery rate; IL: interleukin; IMPACT: Immunological Multi-Parameter Chip Technology; MCP: monocyte chemoattractant protein; PsA: psoriatic arthritis; RA: rheumatoid arthritis; RF: rheumatoid factor; SAM: significance analysis of microarrays; SE: shared epitope; TNF: tumor necrosis factor.

\section{Acknowledgements}

We thank members of the Robinson Laboratory for their scientific discussions and input. This study was funded by NIH NIAMS R21 Al069160, $\mathrm{NIH}$ 1RC1AR058713-01, an American College of Rheumatology Research and Education Foundation Within-Our-Reach Award and Veterans Affairs Health Care System funding to WHR. Roche Diagnostics $\mathrm{GmbH}$ provided the IMPACT assay kits used in this study. COBAS C and TINA-QUANT are trademarks of Roche.

\section{Author details}

'Division of Immunology and Rheumatology, Department of Medicine, Stanford University School of Medicine, Stanford, CA 94305, USA. ${ }^{2}$ Geriatric Research Education and Clinical Centers, Palo Alto VA Health Care System, 3801 Miranda Avenue, Palo Alto, CA 94304, USA. ${ }^{3}$ Roche Diagnostics GmbH, Nonnenwald 2, 82377 Penzberg, Germany. ${ }^{4}$ Department of Dermatology, University of Michigan, Ann Arbor, MI 48109, USA. ${ }^{5}$ Division of 
Rheumatology, The University of Texas Health Science Center at Houston, Houston, TX 77030, USA

\section{Authors' contributions}

PEC, JS, BGH, HE, UK, and WHR designed the studies, performed the studies, analyzed the data, and interpreted the data. JTE and JDR provided the psoriatic arthritis and ankylosing spondylitis patient samples, respectively. TML contributed to data interpretation and provided editorial input. All authors read and approved the final manuscript.

\section{Competing interests}

$\mathrm{BGH}, \mathrm{HE}$, and UK are employees of Roche diagnostics. WHR has served on a scientific advisory board of Roche Diagnostics and has received research and reagent support from Roche Diagnostics for the study of RA biomarkers. Roche Diagnostics owns patents relating to the IMPACT platform. Stanford University has applied for patents related to RA biomarker work performed prior to this study.

Received: 18 December 2010 Revised: 27 April 2011

Accepted: 24 June 2011 Published: 24 June 2011

\section{References}

1. Hueber W, Kidd BA, Tomooka BH, Lee BJ, Bruce B, Fries JF, Sonderstrup G, Monach P, Drijfhout JW, van Venrooij WJ, Utz PJ, Genovese MC, Robinson WH: Antigen microarray profiling of autoantibodies in rheumatoid arthritis. Arthritis Rheum 2005, 52:2645-2655.

2. Hueber W, Tomooka BH, Zhao X, Kidd BA, Drijfhout JW, Fries JF, van Venrooij WJ, Metzger AL, Genovese MC, Robinson WH: Proteomic analysis of secreted proteins in early rheumatoid arthritis: anti-citrulline autoreactivity is associated with up regulation of proinflammatory cytokines. Ann Rheum Dis 2007, 66:712-719.

3. Kievit W, Fransen J, Oerlemans AJ, Kuper HH, van der Laar MA, de Rooij DJ, De Gendt CM, Ronday $\mathrm{KH}$, Jansen TL, van Oijen PC, Brus HL, Adang EM, van Riel PL: The efficacy of anti-TNF in rheumatoid arthritis, a comparison between randomised controlled trials and clinical practice. Ann Rheum Dis 2007, 66:1473-1478.

4. Hueber W, Tomooka BH, Batliwalla F, Li W, Monach PA, Tibshirani RJ, Van Vollenhoven RF, Lampa J, Saito K, Tanaka Y, Genovese MC, Klareskog L, Gregersen PK, Robinson WH: Blood autoantibody and cytokine profiles predict response to anti-tumor necrosis factor therapy in rheumatoid arthritis. Arthritis Res Ther 2009, 11:R76.

5. Claudon A, Vergnaud P, Valverde C, Mayr A, Klause U, Garnero P: New automated multiplex assay for bone turnover markers in osteoporosis. Clin Chem 2008, 54:1554-1563.

6. Monach PA, Hueber W, Kessler B, Tomooka BH, BenBarak M, Simmons BP, Wright J, Thornhill TS, Monestier M, Ploegh H, Robinson WH, Mathis D, Benoist C: A broad screen for targets of immune complexes decorating arthritic joints highlights deposition of nucleosomes in rheumatoid arthritis. Proc Natl Acad Sci USA 2009, 106:15867-15872.

7. Fries JF, Wolfe F, Apple R, Erlich H, Bugawan T, Holmes T, Bruce B: HLADRB1 genotype associations in 793 white patients from a rheumatoid arthritis inception cohort: frequency, severity, and treatment bias. Arthritis Rheum 2002, 46:2320-2329.

8. Bruce B, Fries JF: The Arthritis, Rheumatism and Aging Medical Information System (ARAMIS): still young at 30 years. Clin Exp Rheumatol 2005, 23:S163-167.

9. Arnett FC, Edworthy SM, Bloch DA, McShane DJ, Fries JF, Cooper NS, Healey LA, Kaplan SR, Liang MH, Luthra HS, Medsger TAJ, Mitchell DM, Neustadt DH, Pinals RS, Schaller JG, Sharp JT, Wilder RL, Hunder GG: The American Rheumatism Association 1987 revised criteria for the classification of rheumatoid arthritis. Arthritis Rheum 1988, 31:315-324.

10. Cohen SB, Emery P, Greenwald MW, Dougados M, Furie RA, Genovese MC, Keystone EC, Loveless JE, Burmester GR, Cravets MW, Hessey EW, Shaw T, Totoritis MC: Rituximab for rheumatoid arthritis refractory to anti-tumor necrosis factor therapy: Results of a multicenter, randomized, doubleblind, placebo-controlled, phase III trial evaluating primary efficacy and safety at twenty-four weeks. Arthritis Rheum 2006, 54:2793-2806.

11. Tusher VG, Tibshirani R, Chu G: Significance analysis of microarrays applied to the ionizing radiation response. Proc Natl Acad Sci USA 2001, 98:5116-5121.

12. Tibshirani RJ, Efron B: Pre-validation and inference in microarrays. Stat Appl Genet Mol Biol 2002, 1:Article1.
13. Stone R, Coppock JS, Dawes PT, Bacon PA, Scott DL: Clinical value of ELISA assays for IgM and IgG rheumatoid factors. J Clin Pathol 1987, 40:107-111.

14. Bizzaro N, Mazzanti G, Tonutti E, Villalta D, Tozzoli R: Diagnostic accuracy of the anti-citrulline antibody assay for rheumatoid arthritis. Clin Chem 2001, 47:1089-1093.

15. Ziolkowska M, Kurowska M, Radzikowska A, Luszczykiewicz G, Wiland P, Dziewczopolski W, Filipowicz-Sosnowska A, Pazdur J, Szechinski J, Kowalczewski J, Rell-Bakalarska M, Maslinski W: High levels of osteoprotegerin and soluble receptor activator of nuclear factor kappa B ligand in serum of rheumatoid arthritis patients and their normalization after anti-tumor necrosis factor alpha treatment. Arthritis Rheum 2002, 46:1744-1753.

16. Mullan RH, Matthews $C$, Bresnihan B, FitzGerald O, King L, Poole AR, Fearon $U$, Veale DJ: Early changes in serum type II collagen biomarkers predict radiographic progression at one year in inflammatory arthritis patients after biologic therapy. Arthritis Rheum 2007, 56:2919-2928.

17. Holick MF: Vitamin D deficiency. N Engl J Med 2007, 357:266-281.

18. Romero-Sanchez C, Robinson WH, Tomooka BH, Londono J, Valle-Onate $\mathrm{R}$, Huang F, Deng X, Zhang L, Yang C, Yu DT: Identification of acute phase reactants and cytokines useful for monitoring infliximab therapy in ankylosing spondylitis. Clin Rheumatol 2008, 27:1429-1435.

19. Visvanathan S, Wagner C, Marini JC, Baker D, Gathany T, Han J, van der Heijde D, Braun J: Inflammatory biomarkers, disease activity and spinal disease measures in patients with ankylosing spondylitis after treatment with infliximab. Ann Rheum Dis 2008, 67:511-517.

20. Landewe R: Predictive markers in rapidly progressing rheumatoid arthritis. J Rheumatol Supp/ 2007, 80:8-15.

21. Schellekens GA, Visser $H$, de Jong BA, van den Hoogen FH, Hazes JM, Breedveld FC, van Venrooij WJ: The diagnostic properties of rheumatoid arthritis antibodies recognizing a cyclic citrullinated peptide. Arthritis Rheum 2000, 43:155-163.

22. Nishimura K, Sugiyama D, Kogata Y, Tsuji G, Nakazawa T, Kawano S, Saigo K, Morinobu A, Koshiba M, Kuntz KM, Kamae I, Kumagai S: Meta-analysis: diagnostic accuracy of anti-cyclic citrullinated peptide antibody and rheumatoid factor for rheumatoid arthritis. Ann Intern Med 2007, 146:797-808

23. Teitsson I, Withrington $\mathrm{RH}$, Seifert $\mathrm{MH}$, Valdimarsson H: Prospective study of early rheumatoid arthritis. I. Prognostic value of IgA rheumatoid factor. Ann Rheum Dis 1984, 43:673-678.

24. van der Heijde DM, van Riel PL, van Leeuwen MA, van 't Hof MA, van Rijswijk MH, van de Putte LB: Prognostic factors for radiographic damage and physical disability in early rheumatoid arthritis. A prospective follow-up study of 147 patients. Br J Rheumatol 1992, 31:519-525.

25. Forslind K, Ahlmen M, Eberhardt K, Hafstrom I, Svensson B: Prediction of radiological outcome in early rheumatoid arthritis in clinical practice: role of antibodies to citrullinated peptides (anti-CCP). Ann Rheum Dis 2004, 63:1090-1095.

26. Lee DM, Schur PH: Clinical utility of the anti-CCP assay in patients with rheumatic diseases. Ann Rheum Dis 2003, 62:870-874.

27. Garnero P, Landewe R, Boers M, Verhoeven A, Van Der Linden S, Christgau S, Van Der Heijde D, Boonen A, Geusens P: Association of baseline levels of markers of bone and cartilage degradation with longterm progression of joint damage in patients with early rheumatoid arthritis: the COBRA study. Arthritis Rheum 2002, 46:2847-2856.

28. Combe B, Landewe R, Lukas C, Bolosiu HD, Breedveld F, Dougados M, Emery P, Ferraccioli G, Hazes JM, Klareskog L, Machold K, Martin-Mola E, Nielsen H, Silman A, Smolen J, Yazici H: EULAR recommendations for the management of early arthritis: report of a task force of the European Standing Committee for International Clinical Studies Including Therapeutics (ESCISIT). Ann Rheum Dis 2007, 66:34-45.

29. Morel J, Combe B: How to predict prognosis in early rheumatoid arthritis. Best Pract Res Clin Rheumatol 2005, 19:137-146.

30. Gengenbacher M, Sebald HJ, Villiger PM, Hofstetter W, Seitz M: Infliximab inhibits bone resorption by circulating osteoclast precursor cells in patients with rheumatoid arthritis and ankylosing spondylitis. Ann Rheum Dis 2008, 67:620-624

doi:10.1186/ar3383

Cite this article as: Chandra et al:: Novel multiplex technology for diagnostic characterization of rheumatoid arthritis. Arthritis Research \& Therapy 2011 13:R102. 